\title{
Interval T-S Fuzzy Modeling Based on Minimizing 1-norm on Approximation Error
}

\author{
Xiaoyong Liü,2 \\ College of engineering and technology, Zunyi Normal College, 563006 Zunyi, China \\ E-mail: liuxy2040163.com
}

\section{Shufang Zhou}

Affiliated Hospital of Zunyi Medical College, 563003 Zunyi, China

\section{Zhonggang Xiong}

College of engineering and technology, Zunyi Normal College, 563006 Zunyi, China

As the obtained data in many practical applications tend to be uncertain or inaccurate, the conventional modeling methods characterized by the deterministic model for this type of data have become undesirable. Taking linear programming, the T-S fuzzy model and some ideas from 1-norm minimization into consideration, a novel method identifying interval fuzzy model (INFUMO) consisted of the upper and lower T-S fuzzy model (referred to as $f_{U}$ and $f_{L}$ ) has been studied in this paper. In order to solve the INFUMO, optimization problems based on minimizing 1-norm with respect to the approximation error corresponding to $f_{U}$ and $f_{L}$ are constructed. Finally, the optimization problems are solved by the linear programming and INFUMO is thus constructed. To demonstrate its effectiveness, the proposed method is applied to identify the interval T-S model of static and dynamic nonlinear model with noise. The proposed method can not only deal with uncertain data to be usually modeled as the deterministic model, but also has better robustness.

CENet2017

22-23 July 2017

Shanghai, China

\footnotetext{
${ }^{1}$ Speaker

,2This work is supported by the Youth Fund Project of the Department of Education( No.KY[2016]254,KY[2015]457), by the Joint Fund Project of the Department of Science and Technology of Guizhou Province (No.LH[2016]7003), by the Ph.D Project Foundation of the Zunyi Normal College(No.BS[2015]04), and by the Innovative Talents Plan of Guizhou High-level personnel(No.([2017]19) .
} 


\section{Introduction}

The research of nonlinear system identification based on obtaining a set of data which applies a cost function that approximation error is minimized has caused the extensive concern in the scientific community, especially with the vent of machine learning approaches such as support vector machine and neural network and so on. However, obtained data[1] in many realworld applications usually has some characteristics which are uncertain, imprecise and incomplete. Consequently, modeling method is usually described as fuzzy sets or generalization of the interval data. In order to deal with those information, uncertain modeling methods based on the interval model with regression analysis[2] as a very important tool have been proposed. To analyze a phenomenon in a fuzzy circumstance, a new interval regression analysis based on the regression quantile techniques [3] is used to construct two interval approximation models. To solve these parameters corresponding to the interval regression models, a basic linear programming (LP) problem[4] must be adopted. Obviously, it is difficult that the nonlinear interval regression with LP problem is to determine a nonlinear model from an infinite number of alternatives. Due to the high capability of feedforward neural networks as a universal approximator of nonlinear mappings, some researches $[5,6]$ dealing with the interval data with the interval regression model and a radial basis function network (RBFN)[7] is applied to the interval modeling in the form of regression analysis in the absence of priori information between the input and the output. Meanwhile, aiming to solv the optimization methods which can appropriately seek for good values and the amount of potential node, support vector interval regression networks are applied to the interval regression analysis to avoid these problems by utilizing $\varepsilon$-insensitive support vector regression(SVR)[8].

Considering that T-S (Takagi -Sugeno) fuzzy model[9] has become an important means in modeling the nonlinear static and dynamic system[10] and its approximation accuracy can also be guaranteed[11], it is widely utilized for control and fault detection. The objective of this paper is to present a new interval modeling method. Taking linear programming and TS fuzzy model and some ideas from -norm minimization into consideration, a novel method identifying interval fuzzy model (INFUMO) consisted of the upper and lower T-S fuzzy model (referred to as $f_{U}$ and $f_{L}$ ) has been studied. In order to solve INFUMO, optimization problems based on minimizin 1-norm with respect to approximation error corresponding to $f_{U}$ and $f_{L}$ are constructed. Finally, the optimization problems are solved by linear programming and INFUMO is thus constructed. The proposed method can not only deal with uncertain data to be usually modeled as deterministic model, but also has better robustness.

\section{Interval T-S Fuzzy Modeling Based on Minimizing 1-norm on Approximation Error}

\subsection{Optimization Problem on Approximation Error for INFUMO Using 1-norm}

It is well known that T-S fuzzy model[11] is widely concerned because of the approximation capability modeling nonlinear static and dynamic system. Considering the obtained data

$$
\left(\boldsymbol{x}_{1}, y_{1}\right), \cdots,\left(\boldsymbol{x}_{N}, y_{N}\right) \text {. }
$$

where $\boldsymbol{x}_{\boldsymbol{k}} \in \boldsymbol{R}^{\boldsymbol{n}}$ is the input pattern and $y_{k}$ is the corresponding output. The output meet the requirements corresponding to the nonlinear system.

$$
y_{k}=g\left(\boldsymbol{x}_{\boldsymbol{k}}\right) \text {. }
$$

In term of the Stone Weierstrass condition, there exists a fuzzy system so that

$$
\sup _{\boldsymbol{x}_{k} \in \boldsymbol{R}^{n}}\left|f\left(\boldsymbol{x}_{\boldsymbol{k}}\right)-g\left(\boldsymbol{x}_{\boldsymbol{k}}\right)\right| \text {. }
$$


If the approxiamtion is taken into account, the following error between the real and the fuzzy model outputs is described as,

$$
\lambda_{k}=y_{k}-f\left(\boldsymbol{x}_{k}\right)
$$

To obtain a set of optimal parameters for T-S fuzzy model, the following modeling error is considered,

$$
\min _{\boldsymbol{x}_{k} \in \boldsymbol{R}^{n}}\left\{\lambda_{1}, \lambda_{2}, \cdots, \lambda_{N}\right\} .
$$

Furthermore, this implies that all of the modeling error is to be minimized. In case of the T-S model, parameters identification is composed of two steps. Antecedent structure identification is to determine the membership function which can be solved with a well-known cluster analysis and is usually named as structure identification. After the structure identification is defined, consequent parameters for T-S fuzzy model in (2.6) are to be solved by applying the proposed optimization.

$$
\boldsymbol{P}=\arg \min _{\boldsymbol{P}, \boldsymbol{x}_{\boldsymbol{k}} \in \boldsymbol{R}^{n}}\left|y_{k}-f\left(\boldsymbol{x}_{\boldsymbol{k}}\right)\right|=\arg \min _{\boldsymbol{P}, \boldsymbol{x}_{k} \in \boldsymbol{R}^{n}}\left|y_{k}-\varphi^{T}\left(\boldsymbol{x}_{\boldsymbol{k}}\right) \boldsymbol{x}_{\boldsymbol{k}} \boldsymbol{P}\right| .
$$

or

$$
\boldsymbol{P}=\arg \min _{\boldsymbol{P}, \boldsymbol{x}_{k} \in \boldsymbol{R}^{n}}\left|y_{k}-\sum_{i=1}^{n}\left(\varphi_{i}^{T}\left(\boldsymbol{x}_{\boldsymbol{k}}\right) \boldsymbol{P}_{\boldsymbol{i}}^{\boldsymbol{T}} \boldsymbol{x}_{\boldsymbol{k}}\right)\right| .
$$

where $\boldsymbol{P}_{i}=\left\{p_{0}^{i}, p_{1}^{i}, \cdots, p_{n}^{i}\right\}, \boldsymbol{x}_{i}=\left\{1, x_{1}, \cdots, x_{n}\right\}$.

Following that, the optimization problems corresponding to (2.7) can be translated into a new minimization by introducing 1-norm,

$$
|\lambda|_{1}=\left|\lambda_{1}\right|+\left|\lambda_{2}\right|+\cdots+\left|\lambda_{N}\right| \text {. }
$$

where $\boldsymbol{\lambda}=\left(\lambda_{1}, \lambda_{2}, \cdots, \lambda_{N}\right)$.

INFUMO is consists of the upper and lower T-S fuzzy model (referred to as $f_{U}$ and $f_{L}$ ). In order to solve $f_{U}$, the following constraints should be met.

$$
f_{U}\left(\boldsymbol{x}_{\boldsymbol{k}}\right)-y_{k} \geq 0 \text {. }
$$

Consequently, the optimization problems using (2.7) and (2.9) for $f_{U}$ are described as

$$
\begin{aligned}
\min & \left|\lambda^{U}\right|_{1}=\lambda_{1}^{U}+\lambda_{2}^{U}+\cdots+\lambda_{N}^{U} \\
\text { s.t. } \quad f_{U}\left(\boldsymbol{x}_{\boldsymbol{k}}\right)-y_{k} \leq \lambda_{k}^{U} & \\
& f_{U}\left(\boldsymbol{x}_{\boldsymbol{k}}\right)-y_{k} \geq 0 \quad k=1,2, \cdots, N .
\end{aligned}
$$

Likewise, by replacing the condition $f_{U}\left(\boldsymbol{x}_{\boldsymbol{k}}\right)-y_{k} \geq 0$ in formula (2.10) with $f_{L}\left(\boldsymbol{x}_{\boldsymbol{k}}\right)-y_{k} \leq 0$, corresponding optimization problem is constructed for $f_{L}$.

\subsection{Optimization Problem on Approximation Error for INFUMO Using 1-norm}

For $f_{U}$, the following optimization is solved by the linear programming

$$
\begin{array}{ll}
\min & \left|\boldsymbol{\lambda}^{U}\right|_{1}=\lambda_{1}^{U}+\lambda_{2}^{U}+\cdots+\lambda_{N}^{U} \\
\text { s.t. } & \sum_{i=1}^{n}\left(\varphi_{i}^{T}\left(\boldsymbol{x}_{\boldsymbol{k}}\right) \boldsymbol{P}_{U, i}^{T} \boldsymbol{x}_{\boldsymbol{k}}\right)-y_{k} \leq \lambda_{k}^{U} \\
& \varphi_{i}^{T}\left(\boldsymbol{x}_{\boldsymbol{k}}\right) \boldsymbol{P}_{\boldsymbol{U}, i}^{T} \boldsymbol{x}_{\boldsymbol{k}}-y_{k} \geq 0 \quad k=1,2, \cdots, N .
\end{array}
$$


where $\boldsymbol{P}_{U, i}$ represents the consequent parameter for T-S fuzzy model. Formula (2.11) can be further translated as the following,

$$
\begin{aligned}
& \min \left(\begin{array}{ll}
\mathbf{0}_{(N+1) \times R} & \mathbf{1}_{N \times 1}
\end{array}\right)\left(\begin{array}{c}
\boldsymbol{P}_{U} \\
\Lambda_{U}
\end{array}\right) \\
& \text { s.t } \quad\left(\begin{array}{cc}
-\boldsymbol{\Xi} & \boldsymbol{Z} \\
\boldsymbol{\Xi} & -\boldsymbol{I}
\end{array}\right) \leq\left(\begin{array}{c}
-\boldsymbol{y} \\
\boldsymbol{y}
\end{array}\right) \text {. }
\end{aligned}
$$

where

$$
\begin{gathered}
\boldsymbol{\Xi}=\left(\begin{array}{ccc}
\varphi_{1}\left(\boldsymbol{x}_{\mathbf{1}}\right) \boldsymbol{x}_{\mathbf{1}}^{\boldsymbol{T}} & \cdots & \varphi_{R}\left(\boldsymbol{x}_{\mathbf{1}}\right) \boldsymbol{x}_{\mathbf{1}}^{\boldsymbol{T}} \\
\vdots & \vdots & \vdots \\
\varphi_{1}\left(\boldsymbol{x}_{N}\right) \boldsymbol{x}_{N}^{T} & \cdots & \varphi_{R}\left(\boldsymbol{x}_{N}\right) \boldsymbol{x}_{N}^{T}
\end{array}\right), \boldsymbol{Z}=\mathbf{0}_{N \times N}, \\
\boldsymbol{P}_{U}=\left(P_{U, 1}, \cdots, P_{U, R}\right)^{T}, \Lambda_{U}=\left(\lambda_{1}^{U}, \cdots, \lambda_{N}^{U}\right)^{T}, \boldsymbol{y}=\left(y_{1}, \cdots, y_{N}\right)^{T .}
\end{gathered}
$$

Likewise, for $f_{L}$

$$
\begin{aligned}
& \min \left(\begin{array}{ll}
\mathbf{0}_{(N+1) \times R} & \left.\mathbf{1}_{N \times 1}\right)
\end{array}\right)\left(\begin{array}{l}
\boldsymbol{P}_{L} \\
\Lambda_{L}
\end{array}\right) \\
& \text { s.t } \quad\left(\begin{array}{cc}
-\boldsymbol{\Xi} & \boldsymbol{Z} \\
\boldsymbol{\Xi} & -\boldsymbol{I}
\end{array}\right) \leq\left(\begin{array}{c}
-\boldsymbol{y} \\
\boldsymbol{y}
\end{array}\right) \text {. }
\end{aligned}
$$

One should note that the definition of the existing variables in $f_{L}$ is almost the same as $f_{U}$. The only difference is that the corresponding constraints have changed and there are some variables to be marked by letter L in the form of superscript or subscript. Hence, the introduced variables in formula $f_{L}$ are not explained any further one. by one. By applying linear programming, the optimization problem (2.13) can be solved. As a result, approximation error $\lambda_{k}$ can be also derived and, $f_{L}$ and $f_{U}$ are described respectively,

$$
f_{U}(\boldsymbol{x})=\sum_{i=1}^{n}\left(\varphi_{i}^{T}(\boldsymbol{x}) \boldsymbol{P}_{\boldsymbol{U}, \boldsymbol{i}}^{\boldsymbol{T}} \boldsymbol{x}\right), \quad f_{L}(\boldsymbol{x})=\sum_{i=1}^{n}\left(\varphi_{i}^{T}(\boldsymbol{x}) \boldsymbol{P}_{\boldsymbol{L}, i}^{\boldsymbol{T}} \boldsymbol{x}\right) .
$$

\section{Simulation Results}

The first simple nonlinear function with uncertainty is considered

$$
\begin{gathered}
f(x)=\cos (x) \sin (x), \Delta f(x)=\gamma \cos (8 x) \\
g(x)=f(x)+g(x) .
\end{gathered}
$$

where $\Delta f(x)=\gamma \cos (8 \mathrm{x})$ denotes uncertainty, $0 \leq \gamma \leq 1$. The functions from the class are defined in the domain $S=\{x \mid-1 \leq x \leq 1\}$ and the set of "measurements" is $X=\left\{x_{i}=0.021 \mathrm{k}, k=-47,-46, \cdots, 47\right\} \subset S$. Considering that the dimensionality of the input space is $\mathrm{d}=1$ in this case, the premise and the consequent variables are the same as the measurements. A few values of $\gamma \quad(\gamma=0,0.2,0.4,0.6,0.8)$ for the simple function with uncertainty are described in Figure 1. 


\begin{tabular}{|c|c|c|c|c|c|c|}
\hline \multirow{2}{*}{ RMSE } & \multicolumn{2}{|c|}{$\mathrm{R}=3$} & \multicolumn{2}{c|}{$\mathrm{R}=4$} & \multicolumn{2}{c|}{$\mathrm{R}=5$} \\
\cline { 2 - 7 } & $f_{U}$ & $f_{L}$ & $f_{U}$ & $f_{L}$ & $f_{U}$ & $f_{L}$ \\
\hline $\begin{array}{c}\text { Proposed } \\
\text { method }\end{array}$ & 0.0659 & 0.1111 & 0.0398 & 0.0634 & 0.0290 & 0.0485 \\
\hline Literature[12] & 0.0767 & 0.1169 & 0.0445 & 0.0787 & 0.0379 & 0.0503 \\
\hline
\end{tabular}

Table 1:Comparison Between The Proposed Algorithm and Literature[12].

Two approximation models $f_{L}$ and $f_{U}$ are constructed with the proposed method and the results are shown in Figure. $1(\mathrm{R}=8)$, in which INFUMO is described as $f_{L}$ and $f_{U}$. Meanwhile, RMSEs (root mean squared error) relaed to the two methods are also compared in Table 1. By comparisons, it is clear that the interval model determined by the proposed approach has a very good approximation interval.

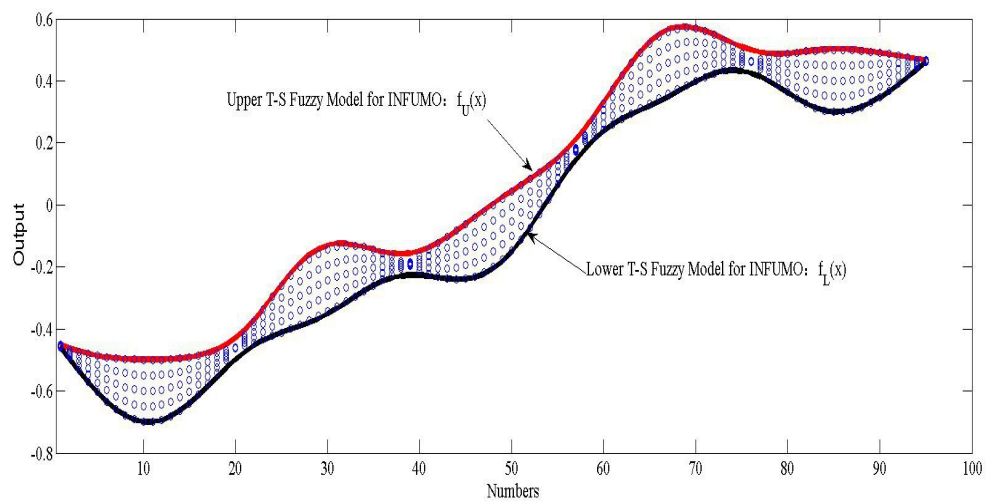

Figure 1: The Proposed Method

Next, the following example involving the nonlinear dynamic system is presented.

$$
y(k+1)=0.5 y(k)+0.5 \frac{y^{2}(k-1)}{1+y^{2}(k-1)}-0.5 y(k-1) u(k)+u(k)
$$

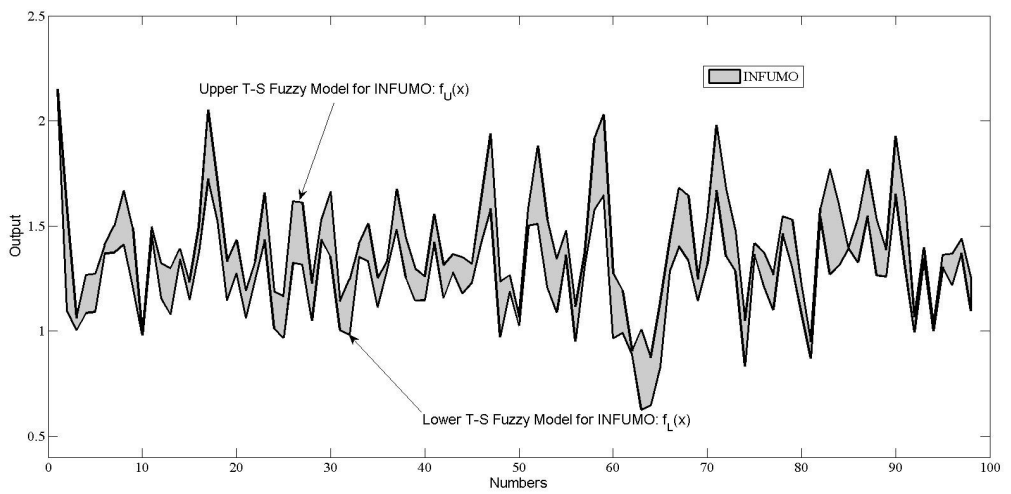

Figure 2 : Interval T-S Fuzzy Model Constructed with The Proposed Method.

where the input signals $u(k)$ are generated randomly and belong to the interval $[0,2]$. One hundred actual data from (2.16) are firstly obtained, and antecedent and consequent variables are chosen as $(u(k-1), y(k-1), y(k-2))$, and $y(k)$ respectively. The 
constructed interval fuzzy model contained all grey area with uncertain data is shown in Figure. 2, which is composed of $f_{L}$ and $f_{U}$. It is indicated in Figure 2 that the proposed method can deal with the asymmetrical interval regression model, which is more suitable for the situation in the presence of uncertain influences.

\section{Conclusion}

In this paper, considering that the obtained data in many practical applications tend to be uncertain or inaccurate, the conventional modeling methods characterized by the deterministic model for this type of data have become undesirable. When a finite set of measurement data is available, a new approach of the interval T-S fuzzy model identification is proposed by combining 1-norm on approximation Error with some ideas from the linear programming theory. Finally, the optimization problems are solved by the linear programming and INFUMO is thus constructed. The proposed method can not only deal with the uncertain data to be usually modeled as the deterministic model, but also has better robustness.

\section{References}

[1] C. Wang, J. Li J, P. Guo P. The normalized interval regression model with outlier detection and its real-world application to house pricing problem[J]. Fuzzy Sets and Systems. 274('”): 109123(2015).

[2] C. Hwang, D. Hong, K. Seok. Support vector interval regression machine for crisp input and output data[J]. Fuzzy Sets and Systems. 157(8):1114-1125(2006).

[3] H. Lee, H. Tanaka H. Upper and lower approximation model s in interval regression using regression quantile techniques[J]. European Journal of Operational Research. 116(3):653666(1999).

[4] H. Tanaka. Fuzzy data analysis by possibilistic linear models[J]. Fuzzy Sets and Systems. 24:363-375 (1987).

[5] H. Kurt. Approximation capabilities of multilayer feedforward networks[J]. Neural Networks. 4: 251C257(1991).

[6] T. Hashiyama, T. Furuhash, Y. Uchikawa Y. An interval fuzzy model using a fuzzy neural .network[C], in: IEEE International Conference Neural Networks. Baltimore, MD, USA. pp. 745-750(1992).

[7] C. Cheng, E. Lee E. Fuzzy regression with radial basis function network[J]. Fuzzy Sets and Systems. 119:291-301(2001).

[8] P. Hao. Interval regression analysis using support vector networks[J]. Fuzzy Sets and Systems. 160(17):2466-2485(2009).

[9] T. Takagi, M. Sugeno. Fuzzy identification of systems and its applications to modeling and control[J]. Systems, Man and Cybernetics,IEEE Transactions on. 1:116-132(1985).

[10] J. Yen, L Wang, C. Gillespie. Improving the interpretability of tsk fuzzy models by combining global learning and locallearning[J]. Fuzzy Systems, IEEE Transactions on. 6(4):530-537 (1998). 
[11] A. Sonbol, M. Fadali, S. Jafarzadeh. Tsk fuzzy function approximators: Design and accuracy analysis[J]. Systems, Man, and Cybernetics, Part B: Cybernetics, IEEE Transactions on. 42(3): 702-712(2012).

[12] I. Skrjanc, S. Blazic, O. Agamennoni. Interval Fuzzy Model Identification Using lo-Norm[J]. IEEE TRANSACTIONS ON FUZZY SYSTEMS. 13(5): 561-568(2005) 